\title{
ERNESTO GARZÓN VALDÉS SOBRE LA SOBERANÍA. UN COMENTARIO
}

\author{
Riccardo Guastini \\ Universidad de Génova
}

RESUMEN. El autor discute un ensayo de EGV sobre los límites jurídicos de la soberanía. EGV distingue dos conceptos de soberano: el soberano-0 (el legislador originario del sistema jurídico) y el soberano-1 (el poder más alto dentro de un sistema jurídico ya establecido). Ahora bien, no tiene sentido hablar de limitaciones jurídicas con referencia al soberano-0, ya que, por definición, sus actos normativos no están reglados por ninguna norma jurídica; en cambio, sí se puede hablar de limitaciones jurídicas del soberano-1, ya que sus actos sí están reglados por normas jurídicas que precisamente lo limitan. RG comparte esta tesis, pero hace tres observaciones. (1) A la misma conclusión podría llegarse analizando los posibles significados de «jurídica» en la expresión "autoridad jurídica suprema»: en un primer sentido, «jurídica» es una autoridad creadora de Derecho; en un segundo sentido, «jurídica» es una autoridad creada por el Derecho. Una autoridad suprema creadora de Derecho no puede tener límites jurídicos, mientras que una autoridad creada por el Derecho, aunque "suprema" (dentro de las autoridades instituidas) no puede no tenerlos. (2) La terminología artificial propuesta por EGV podría ser sustituida por una terminología más simple: los términos usuales «poder constituyente» y «poderes constituidos». (3) El análisis de la soberanía de EGV no puede considerarse completo, porque no abarca el concepto de soberanía del Derecho internacional general, que es precisamente conditio sine qua non de la existencia de obligaciones internacionales, es decir, de limitaciones jurídicas, para los Estados.

Palabras clave: Ernesto Garzón Valdés, soberanía, poder constituyente, poder constituido.

ABSTRACT. The author discusses a paper by EGV concerning the legal limits, if any, of sovereignty. EGV distinguishes two concepts of sovereign: sovereign-0, i.e. the «first» legislator of the legal system at hand, and sovereign-1, i.e. the higher normative power within an already existent legal system. In his view, there is no sense in talking about legal limits as far as the sovereign-0 is concerned, since his normative acts are not governed by any previous legal rule, while the sovereign1 is limited precisely by the legal rules that govern his acts. $R G$ agrees with such a thesis. Nevertheless, he makes three points. (1) The same conclusion could be reached by means of a different strategy, i.e. by analyzing the meanings of «legal» in the context of the phrase «supreme legal authority». In a first sense, a «legal» authority is a law-creating one; in a second sense, a «legal» authority is an authority created by the law. Now, it is a matter of course that a supreme law-creating authority cannot be limited by the law, while any authority created by the law, although "supreme" (within the existent authorities), is necessarily limited by the legal rules by which it was created. (2) The artificial terminology proposed by EGV could be easily substituted by the standard terminology "constituent power» $v$. "constituted powers". (3) EGV's analysis of sovereignty does not apply to international sovereignty of states, since international sovereignty is a conditio sine qua non for the very existence of international obligations, hence legal limits, of states.

Keywords: Ernesto Garzón Valdés, sovereignty, constituent power, constituted power. 


\section{LA TESIS DE ERNESTO GARZÓN VALDÉS}

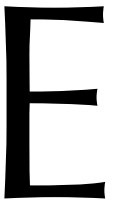

n un ensayo de 1983 sobre los límites jurídicos de la soberanía ${ }^{1}$, Ernesto GARZÓN VALDÉs (en lo que sigue EGV) introduce una importante distinción entre dos conceptos de soberano: en su terminología, el «soberano-cero» y el «soberano-uno».

El soberano-cero no es otra cosa que el soberano sin más (o «autoridad suprema») en el sentido de G. H. vON WRIGHT ${ }^{2}$ : es decir, el agente que realiza el acto normativo (el acto de dictar una norma) que no está reglado por otra norma antecedente de nivel superior, o sea — con las palabras de EGV— «el legislador originario del sistema» jurídico de que se trata.

El soberano-uno, en cambio, es el poder más alto dentro de un sistema jurídico ya establecido: el agente al cual la propia ley (dictada por el legislador originario) atribuye el poder de reglar la conducta social; el legislador, cabe decir, no «originario», sino «derivado».

(Hace falta precisar que, en ambos contextos, se habla de «legislador» y de «ley» no en el sentido formal, sino en el sentido material: en este sentido, «ley» se refiere a cualquier norma general y abstracta, y «legislador» a cualquier agente creador de normas generales y abstractas. En otras palabras, «ley» abarca también las constituciones, así como los reglamentos del ejecutivo; y «legislador» abarca cualquier sujeto con competencia normativa, incluido el poder constituyente) ${ }^{3}$.

Esta distinción permite a EGV sacar una conclusión muy simple: por un lado, no tiene sentido hablar de limitaciones jurídicas con referencia al soberano-cero, ya que, por definición, sus actos normativos no están reglados por ninguna norma jurídica; por otro lado, en cambio, sí se puede hablar de limitaciones jurídicas del soberano-uno, ya que sus actos sí están reglados por normas jurídicas que precisamente lo limitan (y por esta razón dichos actos pueden ser válidos o inválidos).

La distinción de EGV, así como su conclusión, me parece perfecta. Sin embargo, voy hacer algunas observaciones marginales.

\section{DOS TIPOS DE AUTORIDADES «JURÍDICAS»}

Primera observación: me parece que la misma conclusión de EGV podría ser sacada por otra vía, tal vez más simple.

Según la definición estándar — que incluso EGV, al menos prima facie, acepta-el soberano es la «autoridad jurídica suprema». Pero ¿qué quiere decir «jurídica» en este

\footnotetext{
${ }^{1}$ GARZÓN VALDÉs, 1993: «Las limitaciones jurídicas del soberano», en Derecho, ética y política, Madrid: Centro de Estudios Constitucionales, pp. 181 y ss.

${ }^{2}$ VON WRIGHT, 1963: Norm and Action. A Logical Enquiry, London: Routledge \& Kegan Paul, pp. 191 y ss.

${ }^{3}$ Una importante discusión del concepto de ley en sentido material se encuentra en CARRÉ DE MALBERG, 1984: La loi, expression de la volonté générale (1930), París: Nueva Impresión Économica, 1984.
} 
contexto? Esta palabra, en tanto predicado de una autoridad, es ambigua de la forma siguiente:

i) puede denotar una autoridad creadora de Derecho, o bien

ii) una autoridad creada (y reglada) por el Derecho.

Una autoridad suprema que sea «jurídica» en el primer sentido es sencillamente una autoridad de facto — creadora de Derecho, pero no creada por el derecho- cuyas normas son consideradas Derecho vigente no en virtud de otra norma preexistente que le otorgue competencia, sino sobre la base del principio de efectividad ${ }^{4}$. Y la autoridad de facto suprema de un sistema jurídico parece ser, sencillamente, el poder creador de la constitución (o mejor dicho de la «primera» constitución en el caso de una secuencia de constituciones cada una de las cuales está fundada sobre la constitución precedente ${ }^{5}$ ).

Una autoridad «jurídica» en el segundo sentido es cada autoridad instituida por el propio derecho (por medio de normas de competencia), y la autoridad suprema, dentro de las autoridades instituidas, es la autoridad cuyas decisiones no pueden (en sentido deóntico) ser derrotadas por ninguna otra autoridad: en este sentido, por ejemplo, es autoridad suprema el parlamento en algunos sistemas jurídicos, el tribunal constitucional en otros.

La autoridad de facto, siendo de facto, puede por cierto tener límites fácticos, pero no puede lógicamente ser limitada por normas jurídicas: de otra manera, no sería de facto, se convertiría en autoridad «jurídica» en el segundo sentido.

Cualquier autoridad «jurídica» en el segundo sentido, en cambio, sí puede ser jurídicamente limitada por normas — o, mejor dicho, metanormas - de varios tipos: tanto por metanormas (en sentido amplio) sobre el procedimiento normativo, como por metanormas (en sentido estricto) sobre el contenido de las normas futuras.

\section{PODER CONSTITUYENTE Y PODERES CONSTITUIDOS}

Segunda observación: me parece que la misma distinción introducida por EGV podría ser hecha sin necesidad de recurrir a la terminología artificial «soberano-cero» vs. «soberano-uno». Terminología que, por otra parte, no es muy feliz, ya que en un sentido importante el soberano-uno — siendo limitado por normas jurídicas— no es «soberano» en absoluto.

En efecto, ya existe en la teoría constitucional una terminología estándar que permite capturar los dos conceptos de EGV. Me refiero a la oposición poder constituyente vs. poderes constituidos ${ }^{6}$.

Hace falta precisar que en la teoría constitucional se encuentran dos distintos conceptos de poder constituyente, un concepto «formal» y otro «sustancial»:

${ }^{4}$ KelSEn, 1945: General Theory of Law and State, Cambridge (Mass.): Harvard U. P., pp. 118 y ss.

5 KELSEN: pp. 115 y ss.

${ }^{6}$ Como se sabe, la primera formulación de esta distinción se encuentra en SIEYĖs, 1789: Qu'est-ce que le tiers état? Una discusión interesante de varios problemas conectados con el poder constituyente se encuentra en KLEIN, 1996: Théorie et pratique du pouvoir constituant, Paris: PUF. 
i) Desde el punto de vista formal (el de H. KelSEN, por ejemplo), constituyente es un poder no constituido, es decir, no otorgado y reglado por ninguna norma jurídica preexistente, y por tanto fáctico ${ }^{7}$ : «extra ordinem», como se dice en la doctrina constitucional italiana ${ }^{8}$;

ii) Desde el punto de vista sustancial (el de C. SCHMiTT, por ejemplo), constituyente es cualquier poder - fáctico o legal, no importa — que toma la «decisión política fundamental», es decir, elige «los principios supremos del ordenamiento», o «la forma del Estado», o algo por el estilo? .

Ahora bien, es el concepto formal de poder constituyente el que nos interesa aquí.

El soberano-cero no es otra cosa que el poder constituyente en sentido formal.

El soberano-uno es el más alto de los poderes constituidos, es decir, el poder legislativo dentro de un régimen de constitución flexible, el poder de revisión constitucional dentro de un régimen de constitución rígida.

El poder constituyente, siendo no constituido, por definición, no tiene limitaciones jurídicas: como no ha sido otorgado por ninguna norma jurídica, ninguna norma jurídica puede reglarlo. En este sentido, el poder constituyente es soberano. Y por eso no tiene sentido hablar de validez (o de invalidez) de la constitución ${ }^{10}$.

Cualquier poder constituido, en cambio, siendo otorgado por una metanorma jurídica, desde luego puede ser limitado por esta misma o bien por otras metanormas. Y por eso sus normas pueden ser válidas o inválidas.

Sin embargo, dentro de una constitución flexible, el legislador: primero, sólo tiene límites formales relativos al procedimiento legislativo, no límites sustantivos relativos al contenido de las leyes; segundo, en virtud de la flexibilidad de la constitución, tiene incluso el poder de derogar los límites formales, y por tanto de librarse de cualquier límite ${ }^{11}$. En estas circunstancias, el legislador — aun siendo conceptualmente un poder constituido- puede ser considerado «sustancialmente» soberano.

Hace falta decir, sin embargo, que fuera de estas circunstancias (hoy en día muy especiales) el predicado «soberano» referido a cualquier poder constituido es simplemente un mal uso del lenguaje.

\section{EL CONCEPTO DE SOBERANÍA EN DERECHO INTERNACIONAL}

Tercera observación: el análisis de la soberanía hecha por EGV no puede considerarse completo, porque no abarca el concepto de soberanía que se encuentra en el Derecho internacional general (o, mejor dicho, en la doctrina internacionalista: siendo el

${ }^{7}$ KeLSEN, 1945: p. 259.

${ }^{8}$ Por ejemplo: Paladin, 1996: Le fonti del diritto italiano, Bologna: Il Mulino, pp. 16 y ss.

9 SchmitT, 1928: Dottrina della costituzione, trad. it. Milano: Giuffré, 1984, p. 41.

${ }^{10}$ Guastini, 2006: «Jugements de validité», en De Béchillon, D.; Brunet, P.; Champeil-Desplats, V., y Millard, E. (eds.) 2006: L'architecture du droit. Mélanges en l'bonneur du Professeur Michel Troper, Paris, Économica, 2006; GUASTINI, 2004: «Proyecto para la voz "Ordenamiento jurídico" de un diccionario», en Doxa, 27.

${ }^{11}$ Sobre los límites de la legislación dentro de un ordenamiento con constitución flexible resulta fundamental el trabajo de Esposito, 1934: La validità delle leggi. Studio sui limiti della potestà legislativa, i vizi degli atti legislativi e il controllo giurisdizionale, nueva impresión Milano: Giuffré, 1964. 
Derecho internacional general un conjunto de normas consuetudinarias, no hay otra formulación de sus normas que la ofrecida por la doctrina o, a veces, por las convenciones que codifican costumbres preexistentes).

En Derecho internacional: primero, «soberanía» es un predicado no de poderes dentro de un ordenamiento jurídico, sino de estados, es decir precisamente de ordenamientos; segundo, «soberanía» significa no summa potestas — como en Derecho constitucional— sino (más o menos) independencia, en particular, independencia jurídica, un ordenamiento es jurídicamente independiente de cualquier otro ordenamiento si, y solo si, es un ordenamiento «originario», es decir, posee su propia constitución ${ }^{12}$.

Ahora bien, en el Derecho internacional: por un lado, la soberanía —en el sentido que he dicho- es un elemento de la propia definición de «estado» (por definición, un ordenamiento no soberano no es un estado); y, por otro lado, los estados son los (únicos) sujetos del ordenamiento internacional. Dicho de otra forma, la soberanía es uno de los presupuestos para ser destinatarios de normas internacionales.

Evidentemente, en este tipo de contexto, sería un grave error conceptual decir que la soberanía es incompatible con cualquier límite jurídico ${ }^{13}$. Los estados, en tanto soberanos, sí son recíprocamente independientes, pero no son independientes del Derecho internacional ${ }^{14}$. La soberanía de los estados, en tanto presupuesto de la aplicabilidad de normas internacionales, es precisamente la condicio sine qua non de la existencia de obligaciones internacionales, y entonces de limitaciones jurídicas, para los estados. Es decir, la soberanía internacional no excluye, sino implica limitaciones jurídicas ${ }^{15}$.

\footnotetext{
12 Guastini, 2006: Lezioni di teoria del diritto e dello stato, Torino: Giappichelli, 2006, pp. 207 y ss.

13 Es el error de FERRAJOLI, 1995: La sovranità nel mondo moderno. Nascita e crisi dello Stato nazionale, Milano: Anabasi.

${ }^{14}$ KELSEN, 1920: Il problema della sovranità e la teoria del diritto internazionale. Contributo per una dottrina pura del diritto, trad. it. Milano: Giuffré, 1989.

${ }_{16}$ A menos que se sostenga que el ordenamiento internacional no es un ordenamiento «jurídico», lo que, como se sabe, es perfectamente posible.
} 